\title{
Iron deficiency without anaemia is a potential cause of fatigue: meta-analyses of randomised controlled trials and cross-sectional studies
}

\author{
Katsuhiko Yokoi $^{1 *}$ and Aki Konomi ${ }^{2}$ \\ ${ }^{1}$ Department of Human Nutrition, Seitoku University Graduate School, Matsudo, Chiba 271-8555, Japan \\ ${ }^{2}$ Department of Nutritional Sciences, Faculty of Human Ecology, Yasuda Women's University, Hiroshima 731-0153, Japan
}

(Submitted 25 July 2016 - Final revision received 27 March 2017 - Accepted 11 May 2017-First published online 19 June 2017)

\section{Abstract}

Fe deficiency is a prevalent nutritional disease, and fatigue is a common complaint in the general and patient population. The association between Fe deficiency without anaemia (IDNA) and fatigue is unclear. Here, we performed a meta-analysis to evaluate the therapeutic effect of Fe on fatigue in patients with IDNA and the association between IDNA and fatigue in the population. Articles from the PubMed database up to 19 January 2016 were systematically searched. A total of six relevant randomised controlled trials (RCT) and six relevant cross-sectional studies were identified. All outcomes were converted into effect sizes. In the meta-analysis of the six RCT, we identified a significant therapeutic effect of $\mathrm{Fe}$ in fatigue patients with IDNA (pooled effect size $0.33 ; 95 \%$ CI $0 \cdot 17,0 \cdot 48 ; I^{2}=0 \cdot 0 \% ; P<0 \cdot 0001$ ). A sensitivity analysis found that the overall results (i.e. significant association) were robust. In the meta-analysis of the six cross-sectional studies, the association between IDNA and fatigue was not significant (pooled effect size $0 \cdot 10 ; 95 \%$ CI $-0 \cdot 11,0 \cdot 31 ; I^{2}=57 \cdot 4 \% ; P=0 \cdot 362$ ). A sensitivity analysis found that the overall results (i.e. no significant association) were not robust; removal of one study made the outcomes significant. These meta-analyses suggest that improving Fe status may decrease fatigue. Further research is necessary to identify diagnostic criteria for selecting fatigue patients who might benefit from Fe therapy and to assess the prevalence of IDNA with fatigue in the general population.

Key words: Non-anaemic iron deficiency: Iron: Fatigue: Meta-analyses

Fe deficiency is prevalent worldwide. Premenopausal women of reproductive age are at a higher risk for Fe deficiency than other sex/age groups. In the UK National Diet and Nutrition Survey (2008/2009-2011/2012), 15.5\% of women aged 19-64 years were found to have plasma ferritin levels below $15 \mathrm{ng} / \mathrm{ml}^{(1)}$. In the US National Health and Nutrition Examination Survey 1999-2006, 10.9\% of women aged 18-49 years had serum ferritin (sFer) levels below $12 \mathrm{ng} / \mathrm{ml}^{(2)}$. Fe deficiency is more prevalent in Japan than in other developed countries. A total of $36-45 \%$ of women aged 20-29 years and 44-49\% of women aged 30-49 years were Fe-deficient according to the National Health and Nutrition Survey of Japan when a $15 \mathrm{ng} / \mathrm{ml}$ cut-off value for sFer was used ${ }^{(3)}$.

Fe-deficiency anaemia is a well-known cause of functional deterioration, resulting in various symptoms including paleness, fatigue, dyspnoea and headache ${ }^{(4)}$. The seventeenth-century English physician Thomas Sydenham (1642-1689), who first identified the therapeutic effect of Fe, steel and chalybeate (Fe spring water) on 'chlorosis', also used these minerals to treat women's hysteria and men's hypochondria ${ }^{(5)}$. 'Chlorosis' or 'green sickness' corresponds to severe Fe-deficiency anaemia in modern medical terminology. Sydenham seemed to consider 'chlorosis' as a severe condition within a wide spectrum continuing from hysteria and hypochondria, which is close to the concept of neurotic syndrome or neurasthenia in modern medicine ${ }^{(6)}$. In the nineteenth century, there were several case reports (limited to women and children) of non-anaemic chlorosis (i.e. Fe deficiency without anaemia (IDNA) with signs and symptoms including fatigue and dyspnoea $)^{(7,8)}$.

Fatigue not associated with known causes is a very common complaint in the general population ${ }^{(6)}$. In UK surveys, the prevalence of fatigue was $22 \cdot 5 \%^{(9)}$ in the community (16-64 years) and $11.3 \%$ among primary-care patients $(18-45 \text { years })^{(10)}$. About $60-80 \%$ of clinical cases of fatigue had no identified physical cause $^{(10,11)}$. Women complained of more fatigue than men after adjustment for psychological distress in a survey performed in Britain ${ }^{(12)}$. Because women are more likely to be Fe-deficient than men, there could be an association between IDNA and fatigue.

After 1960, there were several randomised controlled trials (RCT) and observational studies regarding the relationship between IDNA and fatigue. The results of RCT on Fe and fatigue

Abbreviations: IDNA, Fe deficiency without anaemia; RCT, randomised controlled trial; sFer, serum ferritin.

* Corresponding author: K. Yokoi, fax +81 47363 1401, email yokoi@seitoku.ac.jp 
are controversial. Beutler et al. ${ }^{(13)}$, Verdon et al. ${ }^{(14)}$, Krayenbuehl et $a l^{(15)}$ and Vaucher et $a l^{(16)}$ found a significant therapeutic effect of Fe on fatigue in patients with IDNA. Morrow et al. ${ }^{(17)}$ and Waldvogel et $a l^{\left({ }^{(1)}\right.}$ did not find a significant association between Fe therapy and fatigue improvement.

The outcomes of cross-sectional studies are also controversial. On the basis of the cross-sectional studies, Piednoir et al. ${ }^{(19)}$, Comin-Colet et al. ${ }^{(20)}$ and Sawada et al. ${ }^{(21)}$ found a positive and significant association between IDNA and fatigue. Lasocki et $a l^{(22)}$ found that IDNA was associated with some increased fatigue scores. Goldenberg et $a l^{(23)}$ did not find an association between IDNA and fatigue. Beck et al. ${ }^{(24)}$ found that IDNA was significantly associated with decreased fatigue, but that this association was no longer statistically significant when factors expected to be associated with $\mathrm{Fe}$ status and fatigue were controlled for. A history of suboptimal Fe status was found to be a significant positive predictor of physical fatigue in their multiple regression model.

Here we aimed to resolve these controversies by performing a systematic review and meta-analysis aimed at determining whether IDNA is associated with fatigue and whether Fe therapy is effective in treating fatigue in patients with IDNA.

\section{Methods}

\section{Literature search and study selection}

A broad literature search was performed using the PubMed database. The preliminary article search was started on 13 August 2015. Articles published from 1809 to January 2016 were identified on 19 January 2016 using the following search formula: Search (((iron deficiency without anaemia) OR (nonanemic OR non-anemic OR non anaemic OR non-anaemic) OR (prelatent iron deficiency OR latent iron deficiency) OR (sideropenia OR sideropaenia OR sideropenic OR sideropaenic)) AND (fatigue OR fatigability OR tiredness)). Searching for 'anaemia' in the PubMed database includes searching for both 'anaemia' and 'anemia'. The study selection process is shown in Fig. 1. An additional search was conducted on 14 February 2016 using the search formula: Search ((iron depletion OR iron-depleted) AND (fatigue OR fatigability OR tiredness)). However, this did not uncover an eligible article except for one duplicate of the initial search.

Exclusion criteria as applied via a screening process included (1) studies based on non-human subjects or on cell cultures; (2) articles published in non-English languages; (3) studies other than RCT or cross-sectional studies; (4) articles that lacked reports on symptomatic fatigue; (5) articles that lacked objective parameters on Fe status or did not include study subjects with IDNA; (6) case reports; (7) perception surveys; and (8) review articles, comments or protocol-only articles and (9) articles with a theme other than Fe deficiency and fatigue. At this stage, articles that lacked an abstract in the PubMed database or that had an abstract not reporting details necessary for exclusion were included and their full-text articles obtained. Inclusion criteria were as follows: (1) cross-sectional studies on symptomatic fatigue between subjects with normal Fe status and those with IDNA or (2) RCT of Fe therapy on symptomatic fatigue in non-anaemic subjects including IDNA. Diagnostic criteria of IDNA were according to the authors of the respective articles. Two investigators independently participated in assessing the eligibility of selected studies. Disagreements were resolved by discussion between the reviewers.

\section{Data extraction and statistical analyses}

The characteristics of the subjects (age, sex, geographical area), study design (cross-sectional study or RCT; parallel or cross-over design only for RCT; type, dosage and duration of Fe preparations

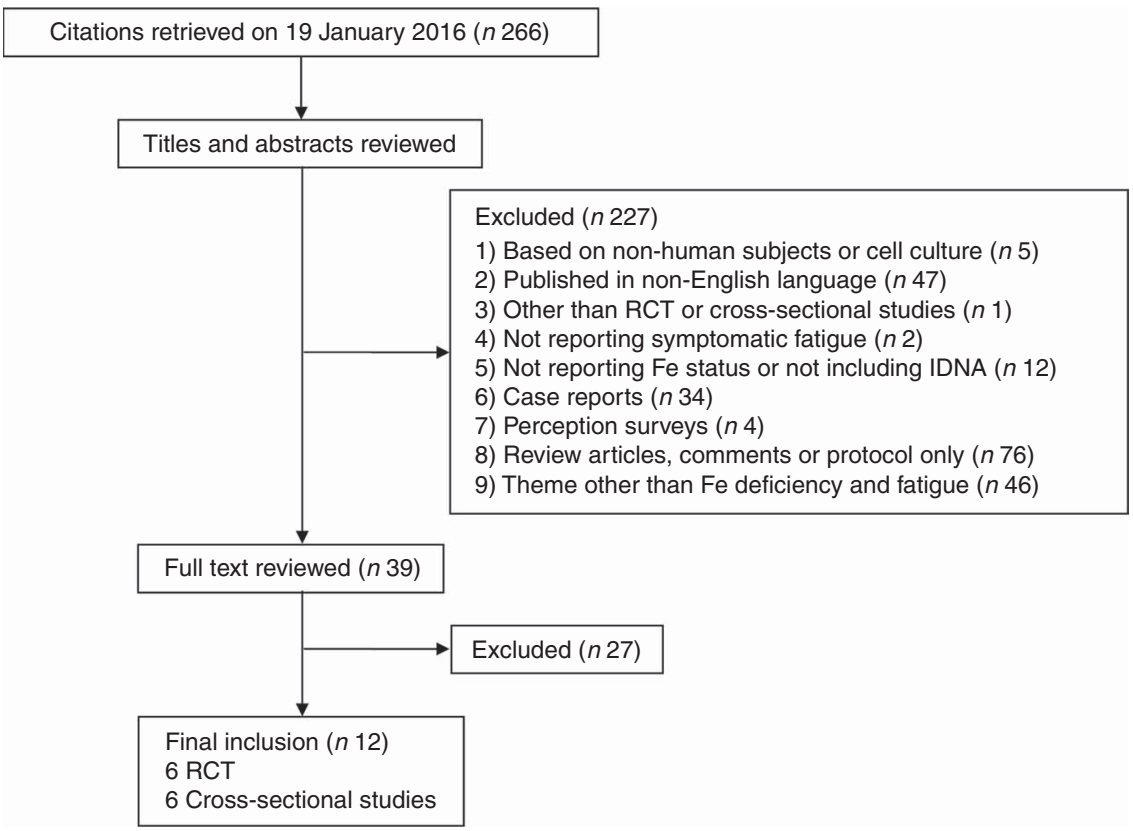

Fig. 1. Flow diagram of the systematic literature search undertaken on iron deficiency without anaemia (IDNA) and fatigue. RCT, randomised controlled trials. 
only for RCT; Fe status parameters; and criteria of Fe status) and measures of the effect sizes (sample sizes; means and standard deviations of fatigue parameters; differences in fatigue parameters between two groups and the corresponding $P$ values; the $P$ value and the regression coefficient corresponding to the specified variable for Fe deficiency in the multivariate linear regression model; the OR and its CI based on the multivariate logistic regression model; the number of parameters for multivariate regression models; the proportion of responders in fatigue treatment by an Fe preparation or placebo; or relative effectiveness in fatigue treatment between the Fe preparation and placebo for a matched comparison design) were extracted.

For cross-sectional studies, Fe status and fatigue parameters determined at the same time were extracted from reports. When the results of multivariate analyses were available, they were also used for meta-analysis.

The conversion of the test statistics into effect sizes $(d)$ and their standard errors $\left(\sigma_{d}\right)$ for the respective study design is shown in the online Supplementary Appendix A. All test statistics were converted into unbiased estimates of standardised mean differences or 'effect size' with their standard errors $^{(25)}$ because of the heterogeneity of symptomatic fatigue parameters $^{(26)}$ and for removal of a small sample bias from standardised mean differences ${ }^{(27)}$. For continuous variables, conversion was carried out as described by Hedges ${ }^{(27)}$. For dichotomous variables, corrected $\log$ OR and corrected standard error for small sample bias were calculated according to the recommendation by $\mathrm{Cox}^{(28)}$. We employed 0.607 of the scale factor in the conversion of $\log$ OR to effect size for adjusting the logistic distribution to the normal distribution originally recommended by $\mathrm{Cox}^{(28)}$, rather than division by its reciprocal number $1.65^{(29)}$. Although Sanchez-Meca et al. estimated the bias of the effect-size indices in the unmatched design using the Monte Carlo simulation ${ }^{(29)}$, they did not analyse the matched-pair design. Then, we performed a simulation study to estimate the bias of Haldane's corrected $\log \mathrm{OR}^{(30)}$ and that of the corrected standard error by Gart \& Zweifel ${ }^{(31)}$ recommended by $\mathrm{Cox}^{(28)}$, which were found to be negligible (online Supplementary Appendix B). For the multivariate linear regression model, conversion was carried out as described by Borenstein ${ }^{(32)}$ after calculating the corresponding partial correlation coefficient from $P$ values determined by the partial $F$ test $^{(33)}$ (online Supplementary Appendix A).

If the overall score and its subscores were reported, the result of the overall score was taken as the representative value of the study. If multiple measures were reported in a single study, a simple average of effect sizes was calculated as a representative value. The corresponding standard error was calculated as the square root of the weighted average of squared standard errors using the degree of freedom (sample size minus 1) as weights.

Meta-analysis of effect sizes was performed using $\mathrm{R}$ version 3.2.2 and the meta-analysis package 'metafor' version 1.9-8 with DerSimonian-Laird weights in a random-effects model. The results were summarised as the effect sizes, standard errors and $95 \%$ CI. The significance of the pooled effect size was determined by a $Z$ test. $P<0.05$ was considered to be statistically significant.
The heterogeneity within the outcomes of the studies was evaluated using the $I^{2}$ statistics. Sensitivity analysis was also performed with one study removed at a time to evaluate whether a single study affected the outcomes. Potential publication bias was assessed by funnel plots displaying standard error and effect size.

\section{Results}

\section{Study selection and characteristics}

A total of 266 citations were initially retrieved by a search of the PubMed database. Finally, twelve studies were included for further analyses. The total number of participants was 1875 and the number of females was 1344 (71.7\%). In all, six RCT and six cross-sectional studies were selected. The study populations originated from the following geographical areas: France $^{(16,19,22)}$, Switzerland ${ }^{(14,15,18)}$, Canada ${ }^{(23)}$ Japan $^{(21)}$, New Zealand $^{(24)}$, Spain ${ }^{(20)}$, the $\mathrm{UK}^{(17)}$ and the $\mathrm{USA}^{(13)}$.

The characteristics of the participants (age, country of origin, feature of the population, proportion of women and sample size), inclusion criteria of the participants, definition of $\mathrm{Fe}$ deficiency, mean $\mathrm{Hb}$ and sFer levels, study design (parallel-arm or cross-over, only for RCT) and description of Fe treatment (only for RCT) are shown in Table 1 (for the RCT) and Table 2 (for the cross-sectional studies). The fatigue scale, outcome, and corresponding effect size and standard errors, including the results for multiple measures, if present, are described in the online Supplementary Appendices C (for the RCT), $\mathrm{D}$ (for the cross-sectional studies using univariate analysis) and E (for the cross-sectional studies using multivariate analysis).

All six RCT were double-blind, placebo-controlled and exclusively focused on younger adult women ${ }^{(13-18)}$. Two studies employed a cross-over design, and four used a parallelarm design. In five trials, the study population comprised patients reporting fatigue or having symptoms mainly of fatigue (Table 1). In one Swiss study, blood donors who visited the Red Cross Transfusion Centre participated in the trial ${ }^{(18)}$. One trial used intravenous $\mathrm{Fe}$ and the other five trials used oral preparations.

Two cross-sectional studies targeted young women aged about 20 years from the general population. The New Zealand study was a community-based study without subject selection by inclusion and exclusion criteria ${ }^{(24)}$. In this study, multivariate analysis was used to correct for confounders, because the characteristics of the IDNA and non-ID groups in the population were quite different ${ }^{(24)}$. In the Japanese study, the participants were screened by inclusion and exclusion criteria for intervention trials $^{(21)}$. In this study, the characteristics of the IDNA and non-ID groups were well-matched and multivariate analysis was not performed ${ }^{(21)}$

The other four studies focused on patients with chronic heart failure or inflammatory bowel disease and those who had undergone cardiac surgery or had been admitted to intensive care units (Table 2). The design of these four clinical studies was a cross-sectional comparison of IDNA and non-ID patients in longitudinal observation. In addition to sFer, all four clinical studies employed serum transferrin receptor (sTfR) and/or 


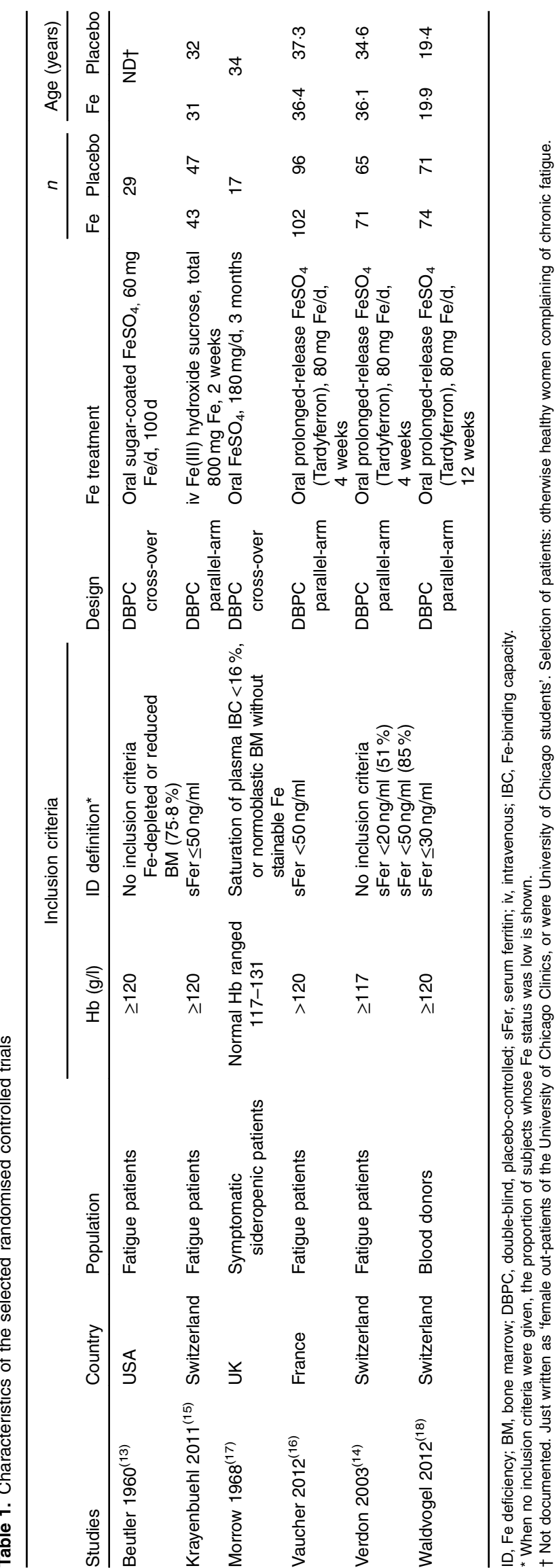

transferrin saturation (TS) as an Fe parameter insensitive to inflammation, a confounding factor causing elevated sFer and anaemia in chronic disease ${ }^{(34)}$, as well as fatigue ${ }^{(35)}$. In the study by Piednoir et $a l .{ }^{(19)}$, serum C-reactive protein (CRP) combined with sTfR:log ferritin ratio was also employed in the diagnosis of ID, and no significant difference in CRP was found between the IDNA and non-ID groups. In their study, multivariate analysis was not performed.

\section{Meta-analysis and sensitivity analysis for randomised controlled trials}

The selected studies used various fatigue assessment scales and evaluation methods for outcomes including continuous and dichotomous data. Therefore, all outcomes were converted to effect sizes. The results of the meta-analysis for RCT are shown in Fig. 2. The pooled effect size in the meta-analysis of the RCT was highly significant, indicating the effectiveness of Fe treatment to relieve fatigue in non-anaemic subjects, principally IDNA. By removing one study at a time, the $I^{2}$ statistics changed from 0.0 to 11.8 and the $P$ value barely changed (from $<0.0001$ to 0.0022). The funnel plot for the meta-analysis on the RCT is shown in Fig. 3 and is not suggestive of publication bias.

\section{Meta-analysis and sensitivity analysis for cross-sectional studies using outcomes from univariate analysis}

The results of cross-sectional studies on the IDNA-fatigue relationship based on outcomes from the univariate analysis are described in Fig. 4. In the meta-analysis of the cross-sectional studies, heterogeneity was evident $\left(I^{2}=49 \cdot 5 \%\right)$. The effect size of the study by Beck et al. ${ }^{(24)}$ was negative, corresponding to the finding that women with IDNA reported fatigue less often than women with normal Fe status in their study population.

Removing the study by Beck et al. ${ }^{(24)}$ reduced the heterogeneity among the studies to insignificance $\left(I^{2}=0.0 \%\right)$, although the removal of any other study kept $I^{2}$ values above $50 \%$. Therefore, heterogeneity was considered to be attributable to the study by Beck et al. ${ }^{(24)}$. By excluding the Beck et al. study, the effect becomes significant. The funnel plot for the meta-analysis on the cross-sectional studies is shown in Fig. 5, which suggests that there could be unpublished studies in the left part of the funnel. Also, there might be two subpopulations with different effect sizes. However, the number of studies in the current data set is insufficient to test this possibility.

\section{Meta-analysis and sensitivity analysis for cross-sectional studies using outcomes from multivariate analysis}

The severity of inflammatory diseases presumably affected the outcomes of clinical studies. Therefore, effect sizes corrected for confounders by multivariate analysis were used in our metaanalysis, if they were present. The results of our meta-analysis are shown in Fig. 6. Compared with meta-analysis using effect sizes based on univariate analysis, the overall effect size was smaller in the meta-analysis using outcomes from multivariate analyses, whereas the general tendency was similar to that 
Table 2. Characteristics of the selected cross-sectional studies (Numbers and percentages)

\begin{tabular}{|c|c|c|c|c|c|c|c|c|c|c|c|c|c|c|}
\hline \multirow[b]{3}{*}{ Studies } & \multirow[b]{3}{*}{ Country } & \multirow[b]{3}{*}{ Population } & \multirow[b]{3}{*}{ Inclusion $\mathrm{Hb}(\mathrm{g} / \mathrm{l})$} & \multirow[b]{3}{*}{ Definition of ID } & \multicolumn{4}{|c|}{ Female } & \multirow{2}{*}{\multicolumn{2}{|c|}{$\begin{array}{l}\text { Mean age } \\
\text { (years) }\end{array}$}} & \multirow{2}{*}{\multicolumn{2}{|c|}{ Mean $\mathrm{Hb}(\mathrm{g} / \mathrm{l})$}} & \multirow{2}{*}{\multicolumn{2}{|c|}{$\begin{array}{l}\text { Mean sFer } \\
(\mathrm{ng} / \mathrm{ml})\end{array}$}} \\
\hline & & & & & \multicolumn{2}{|c|}{ IDNA } & \multicolumn{2}{|c|}{ non-ID } & & & & & & \\
\hline & & & & & $n$ & $\%$ & $n$ & $\%$ & IDNA & non-ID & IDNA & non-ID & IDNA & non-ID \\
\hline Beck 2012 ${ }^{(24)}$ & New Zealand & $\begin{array}{l}\text { Unscreened population in } \\
\text { the communities }\end{array}$ & $\mathrm{Hb} \geq 120$ & $\mathrm{sFer}<20 \mathrm{ng} / \mathrm{ml}$ & 22 & 100 & 211 & 100 & 21 & 23 & 131 & 135 & 13 & 47 \\
\hline Comin-Colet $2013^{(20)}$ & Spain & $\begin{array}{l}\text { Baseline data of a CHF- } \\
\text { patient cohort }\end{array}$ & $\mathrm{Hb} \geq 85^{*}$ & $\begin{array}{l}\text { sFer }<100 \mathrm{ng} / \mathrm{ml} \text { or } \\
\text { TS }<20 \% \text { when sFer is } \\
<800 \mathrm{ng} / \mathrm{ml}\end{array}$ & 349 & 46 & 203 & 38 & 73 & 70 & 123 & 131 & $82 \dagger$ & $232 \dagger$ \\
\hline Goldenberg $2013^{(23)}$ & Canada & $\begin{array}{l}\text { Baseline data of an IBD- } \\
\text { patient cohort }\end{array}$ & $\begin{array}{l}\text { Male } \mathrm{Hb} \geq 140 \\
\text { female } \mathrm{Hb} \geq 120\end{array}$ & $\begin{array}{l}\mathrm{sFer}<20 \mathrm{ng} / \mathrm{ml} \text { or } \\
\mathrm{sTfR}>28 \mathrm{mg} / \mathrm{l}\end{array}$ & \multicolumn{2}{|c|}{$39 \ddagger$} & \multicolumn{2}{|c|}{$191 \ddagger$} & \multicolumn{2}{|c|}{44} & \multicolumn{2}{|c|}{ Non-anaemic§ } & $-\S$ & $-\S$ \\
\hline Lasocki 2014 ${ }^{(22)}$ & France & $\begin{array}{l}\text { Data on day } 28 \text { after ICU } \\
\text { discharge from an ICU- } \\
\text { patient cohort }\end{array}$ & $\begin{array}{l}\text { Male } 100<\mathrm{Hb}<130 \\
\text { female } 100<\mathrm{Hb} \\
<120\end{array}$ & $\begin{array}{l}\text { sFer }<20 \mathrm{ng} / \mathrm{ml} \text { or } \\
\text { sFer }<300 \mathrm{ng} / \mathrm{ml} \text { and } \\
\text { TS }<20 \%\end{array}$ & \multicolumn{2}{|c|}{$20 \|$} & \multicolumn{2}{|c|}{$60 \|$} & 64 & 59 & 115 & 113 & 207 & 522 \\
\hline Piednoir $2011^{(19)}$ & France & $\begin{array}{l}\text { Baseline (or day } 0 \text {, that is, } \\
\text { before surgery) data from } \\
\text { a CS-patient cohort }\end{array}$ & See captions $\mathbb{\|}$ & $\begin{array}{l}\text { sFer }<80 \mathrm{ng} / \mathrm{ml} \text { or } \\
\text { sFer } 80-150 \mathrm{ng} / \mathrm{ml} \text { and TS } \\
<20 \% \text { and CRP }<5 \mathrm{mg} / \mathrm{l} \\
\text { or CRP } \geq 5 \mathrm{mg} / \mathrm{l} \text { and sTfR:log } \\
\text { ferritin ratio }>0.7\end{array}$ & 37 & 51 & 63 & 21 & 63 & 70 & 128 & 137 & 53 & 186 \\
\hline Sawada $2014^{(21)}$ & Japan & $\begin{array}{l}\text { Screened participants for } \\
\text { intervention trials }\end{array}$ & $\mathrm{Hb} \geq 120$ & sFer $<20 \mathrm{ng} / \mathrm{ml}$ & 29 & 100 & 36 & 100 & 19.9 & 19.4 & 129 & 133 & $13 \cdot 0$ & 38.7 \\
\hline
\end{tabular}

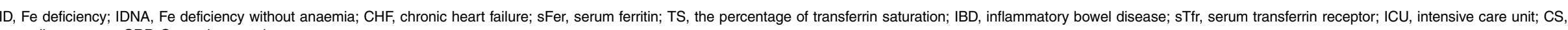
cardiac surgery; CRP, C-reactive protein.

* Exclusion criteria was set for $\mathrm{Hb}$ concentration $(<85 \mathrm{~g} / \mathrm{l})$ in the article by Comin-Colet (2013).

$\dagger$ Median is shown because no mean value is reported.

$¥ 62 \%$ female in the total subjects.

$\S$ No values for $\mathrm{Hb}$ concentration and sFer are given.

II Although $33 \%$ of nine ID patients were female and $21 \%$ of ninety-eight non-ID patients were female at ICU discharge, the proportion of females were not reported on day 28 after ICU discharge.

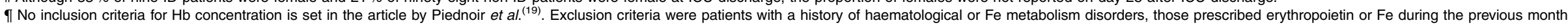




\begin{tabular}{|c|c|c|c|c|c|c|c|}
\hline Study & $\mathrm{ES}^{*}(95 \% \mathrm{Cl})$ & & & SE & $\%$ Weight $^{\dagger}$ & $P_{(i)}^{\ddagger}$ & $I_{(i)}^{2}{ }^{\ddagger}$ \\
\hline Vaucher 2012 & $0.38(0.10,0.66)$ & & -1 & $0 \cdot 14$ & $30 \cdot 7$ & 0.0022 & $8 \cdot 6$ \\
\hline Waldvogel 2012 & $0.06(-0.27,0.38)$ & & 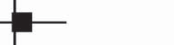 & $0 \cdot 17$ & $22 \cdot 6$ & $<0.0001$ & 0.0 \\
\hline Verdon 2003 & $0.51(0.17,0.85)$ & & - & 0.17 & $20 \cdot 9$ & 0.0016 & 0.0 \\
\hline Krayenbuehl 2011 & $0.49(0.02,0.96)$ & & & 0.24 & $11 \cdot 0$ & 0.0002 & 0.4 \\
\hline Morrow 1968 & $0.14(-0.66,0.94)$ & & - & 0.41 & $3 \cdot 7$ & 0.0001 & $7 \cdot 3$ \\
\hline Beutler 1960 & $0.31(-0.15,0.77)$ & & $\rightarrow-$ & 0.24 & $11 \cdot 1$ & 0.0003 & $11 \cdot 8$ \\
\hline \multirow[t]{3}{*}{ Pooled results } & $0.33(0.17,0.48)$ & & & 0.08 & $Z=4 \cdot 16$ & $P<0.0001$ & $I^{2}=0.0$ \\
\hline & & $-1.0-0.5$ & $0.5 \quad 1.0$ & & & & \\
\hline & & $\begin{array}{l}\mathrm{Fe} \\
\text { increases } \\
\text { fatigue }\end{array}$ & $\begin{array}{r}F e \\
\text { decreases } \\
\text { fatigue }\end{array}$ & & & & \\
\hline
\end{tabular}

Fig. 2. Meta-analysis of the randomised controlled trials on the therapeutic effect of iron on fatigue. ES, effect size. * The positive sign signifies that iron treatment is effective to reduce fatigue. $\dagger 1 / \mathrm{SE}^{2}$ is used as a weight. $¥$ The ith study is excluded from the model-fitting.

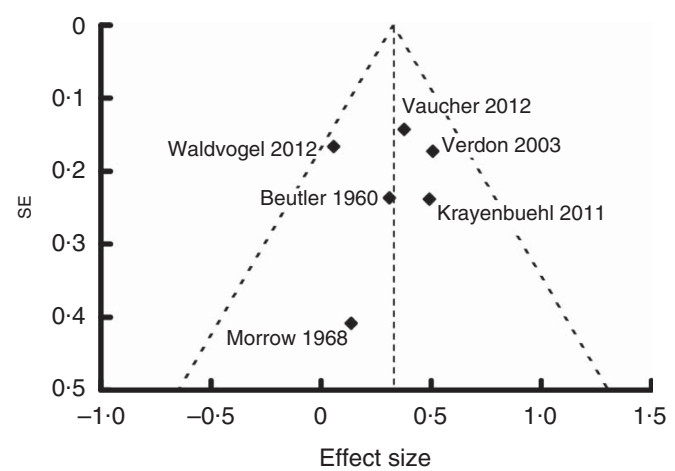

Fig. 3. Funnel plot of the meta-analysis of the randomised controlled trials.

obtained from the univariate analysis. Similar to the metaanalysis based on outcomes of univariate analysis, heterogeneity was evident $\left(I^{2}=57 \cdot 4 \%\right)$. Our sensitivity analysis found that the heterogeneity was introduced by the Beck et al. ${ }^{(24)}$ study. The association between IDNA and fatigue became significant after excluding this study. Publication bias was not evident in the funnel plot for effect sizes based on the multivariate analysis (Fig. 7).

\section{Discussion}

On the basis of a narrative review, Agarwal proposed potential non-haematological benefits of Fe supplementation that include improvements in immune competence, physical function, restless-leg syndrome and cognition ${ }^{(36)}$. Recently, Pratt \& Khan $^{(37)}$ reported a meta-analysis based on twenty-one studies that analysed the relationship between IDNA and various physiological outcomes. Regarding fatigue, Pratt \& Khan only included the study by Krayenbuehl et al. ${ }^{(15)}$ and a meta-analysis was not possible. This was probably due to the strict eligibility criteria, that is, IDNA defined as sFer $<16 \mathrm{ng} / \mathrm{ml}(<12 \mathrm{ng} / \mathrm{ml}$ if age $<5$ years) in the absence of anaemia. Because more relaxed criteria (i.e. IDNA defined according to the authors' own criteria) were used in our study, twelve studies related to this issue were identified. A variety of thresholds were used for sFer and TS to detect Fe deficiency in various countries around the world $^{(38)}$. The recent trend is to use a higher threshold for sFer than has been applied previously ${ }^{(3)}$, especially after Mast et al. found that $30 \mathrm{ng} / \mathrm{ml}$ of sFer gave higher sensitivity and maintained high specificity compared with $12 \mathrm{ng} / \mathrm{ml}$ of $\mathrm{sFer}^{(39)}$. Thus, to include as many relevant studies as possible, it is now considered reasonable to accept the authors' own criteria for detecting Fe deficiency, rather than strictly following the World Health Organization recommendations ${ }^{(40)}$.

By the meta-analysis of the six RCT, a significant treatment effect of $\mathrm{Fe}$ on fatigue was revealed in IDNA patients with fatigue. Because we accepted various authors' thresholds of sFer to define IDNA, it is not possible to know the threshold effective in finding fatigue patients who benefit from Fe treatment. Large clinical trials are recommended to determine the threshold of sFer to select those fatigue patients who might benefit from Fe therapy. Alternatively, because IDNA is prevalent in women, the use of an Fe preparation or improvement in Fe status through proper diet should be considered for nonanaemic women with fatigue.

Because effect sizes for RCT are not intuitive and somewhat difficult to interpret ${ }^{(41)}$, the effect size $(d)$ found by the metaanalysis for Fe therapy for fatigue in IDNA patients with fatigue was converted to an OR using the method of Hasselblad \& Hedges $^{(42)}$, that is, $e^{d \pi / \sqrt{ } 3}$. The effect size for Fe therapy for fatigue was 0.33 and the corresponding OR was 1.8. In the fatigue therapy for cancer patients, large placebo effects were reported $^{(43,44)}$. Considering the relatively large placebo effect, an OR of 1.8 is rather small but clinically meaningful.

In the meta-analysis of cross-sectional studies, the overall effect size became smaller after correction for confounders by multivariate analysis. Significant covariates included CRP, Hb $(<10 \mathrm{~g} / \mathrm{dl})$, active disease, current medical condition and other factors associated with active diseases or with medical condition (Fig. 6). Correction by multivariate analysis is considered 


\begin{tabular}{|c|c|c|c|c|c|c|}
\hline Study & $\mathrm{ES}^{*}(95 \% \mathrm{Cl})$ & & SE & $\%$ Weight $^{\dagger}$ & $P_{(i)}^{\ddagger}$ & $I_{(i)}^{2}{ }^{\ddagger}$ \\
\hline Comin-Colet 2013 & $0.30(0.09,0.51)$ & & $0 \cdot 11$ & $26 \cdot 5$ & 0.482 & $49 \cdot 1$ \\
\hline Goldenberg 2013 & $0.09(-0.27,0.46)$ & & 0.19 & $18 \cdot 0$ & 0.254 & $58 \cdot 5$ \\
\hline Piednoir 2011 & $0.09(-0.31,0.50)$ & & 0.21 & $16 \cdot 0$ & 0.245 & $58 \cdot 7$ \\
\hline Beck 2012 & $-0.39(-0.83,0.05)$ & & 0.22 & $14 \cdot 6$ & 0.001 & 0.0 \\
\hline Sawada 2014 & $0.51(0.02,1.01)$ & - & 0.25 & $12 \cdot 7$ & 0.408 & $50 \cdot 1$ \\
\hline Lasocki 2014 & $0.25(-0.25,0.76)$ & & 0.26 & $12 \cdot 2$ & 0.313 & $59 \cdot 3$ \\
\hline \multirow[t]{3}{*}{ Pooled results } & $0.15(-0.07,0.37)$ & & 0.11 & $Z=1 \cdot 34$ & $P=0 \cdot 181$ & $I^{2}=49 \cdot 5$ \\
\hline & $\begin{array}{cc}1 \\
-1 \cdot 0 & -0.5\end{array}$ & $\begin{array}{ll}1 \\
0.5 & 1.0\end{array}$ & & & & \\
\hline & $\begin{array}{l}\text { Lower } \\
\text { fatigue } \\
\text { in IDNA }\end{array}$ & $\begin{array}{r}\text { Higher } \\
\text { fatigue } \\
\text { in IDNA }\end{array}$ & & & & \\
\hline
\end{tabular}

Fig. 4. Meta-analysis of the cross-sectional studies using outcomes from univariate analysis on the association between iron deficiency without anaemia (IDNA) and fatigue. ES, effect size; ID, iron deficiency. ${ }^{*}$ The positive sign signifies that subjects in the IDNA group complain of more fatigue than those in the non-ID group. $\dagger 1 / \mathrm{sE}^{2}$ is used as a weight. $¥$ The ith study is excluded from the model-fitting.

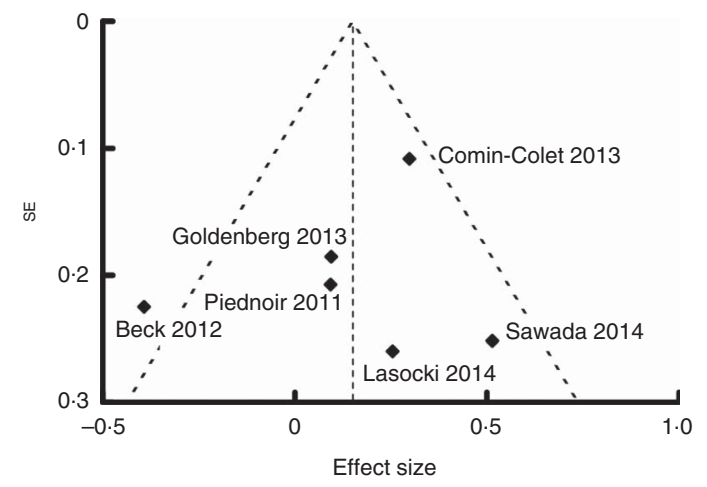

Fig. 5. Funnel plot of the meta-analysis of the cross-sectional studies using outcomes from univariate analysis.

necessary for observational studies unscreened by these criteria or unmatched for these variables. In the meta-analysis of the cross-sectional studies, heterogeneity was relatively large. The pooled effect size in the cross-sectional studies was positive but not significant. From the sensitivity study on the six crosssectional studies, heterogeneity was found to be introduced by the one study based on an unscreened population from the communities. Exclusion of this study made the association between IDNA and fatigue significant, whereas the remaining five cross-sectional studies were based on a specific population (i.e. screened participants for intervention trials or patients with certain disease) (Table 2). More community-based studies will be needed to determine whether the association between IDNA and fatigue also exists in the general population. The RCT focused on a subset of patients with IDNA whose chief complaint was fatigue, whereas the cross-sectional studies surveyed the fatigue level of the subjects with IDNA or without Fe deficiency. A stronger association between $\mathrm{Fe}$ and fatigue should, therefore, be expected in the RCT than in the crosssectional studies.

It is not clear how IDNA causes fatigue. One possible mechanism for this is that decreased $\mathrm{VO}_{2}$ by tissues, especially muscles, in IDNA creates cardiopulmonary stress, evoking a feeling of fatigue. Brownlie et al. ${ }^{(45)}$ found that Fe supplementation increased $\mathrm{VO}_{2 \max }$ in untrained women with IDNA. Brutsaert et al. ${ }^{(46)}$ found that Fe supplementation significantly improved muscle fatigability in young women with IDNA. Another possible mechanism is that Fe deficiency directly affects brain function (neither via a change of $\mathrm{Hb}$ nor peripheral $\mathrm{Fe}$ ) and leads to a feeling of fatigue. Animal studies present evidence supporting this hypothesis. Rats exposed to a marginal Fe diet $(20 \mathrm{mg} / \mathrm{kg})$ through gestation and weaning exhibited abnormal auditory brainstem responses ${ }^{(47)}$. Marginally Fe-deficient rats without decreased $\mathrm{Hb}$ showed abnormal behaviour (i.e. increased activity and stereotypical behaviour in a light cycle), implying abnormality in dopaminergic neurons of the brain ${ }^{(48)}$. Observational studies also found a significant association of IDNA with human psychology and behaviour. Sawada et al. $^{(21)}$ reported that women with IDNA had higher anger and tension in addition to increased fatigue. Lozoff et $a{ }^{\left({ }^{(49)}\right.}$ reported that infants with Fe deficiency (with or without anaemia) had unfavourably altered social-emotional behaviour. Verbal learning and memory function were significantly improved by Fe supplementation in adolescent girls with IDNA ${ }^{(50)}$. These studies support the idea that Fe deficiency directly affects human brain function independently of $\mathrm{Hb}$ levels in the blood.

\section{Strengths and limitations}

This is the first meta-analysis of RCT and cross-sectional studies focused on IDNA and fatigue. The strength of this study is that all studies published and recorded in PubMed from 1809 to 2015 were included. Compared with the existing meta-analysis on IDNA $^{(37)}$, the inclusion criteria were more relaxed and thus included more eligible studies. It would appear that this relaxation is necessary to accommodate diversity in the diagnostic criteria of IDNA. Another merit of the current study is the use of effect size, which enabled the performance of a metaanalysis by using diversified psychological tests for fatigue. 


\begin{tabular}{|c|c|c|c|c|c|c|c|c|}
\hline Study & $\mathrm{ES}^{*}(95 \% \mathrm{Cl})$ & & & SE & $\%$ Weight $^{\dagger}$ & $P_{(i)}^{\ddagger}$ & $I_{(i)}^{2}{ }^{\ddagger}$ & Significant covariate \\
\hline Comin-Colet 2013 & $0.22(0.05,0.40)$ & & & 0.09 & $26 \cdot 1$ & 0.660 & $51 \cdot 5$ & Age, CRP and other eight covariates $\S$ \\
\hline Goldenberg 2013" & $0.07(-0.33 \quad 0.48)$ & & & 0.21 & $14 \cdot 6$ & 0.409 & $65 \cdot 8$ & Presence of active disease \\
\hline Piednoir 2011 & $0.09(-0.31,0.50)$ & & & 0.21 & 14.5 & 0.426 & $65 \cdot 9$ & None ${ }^{\pi}$ \\
\hline Beck 2012 & $-0.26(-0.52,0.01)$ & & & $0 \cdot 14$ & $20 \cdot 5$ & 0.003 & 0.0 & Smoker and other three covariates ${ }^{* *}$ \\
\hline Sawada 2014 & $0.51(0.02,1.01)$ & & - & 0.25 & $11 \cdot 6$ & 0.680 & $55 \cdot 4$ & $\mathrm{None}^{\dagger \dagger}$ \\
\hline Lasocki 2014 & $0.08(-0.39,0.56)$ & & - & $0 \cdot 24$ & $12 \cdot 2$ & 0.411 & $65 \cdot 9$ & Age (>60 years) and $\mathrm{Hb}(<10 \mathrm{~g} / \mathrm{dl})$ \\
\hline \multirow[t]{3}{*}{ Pooled results } & $0.10(-0.11,0.31)$ & & & $0 \cdot 11$ & $Z=0.91$ & $P=0.362$ & $I^{2}=57 \cdot 4$ & \\
\hline & $-1 \cdot 0$ & -0.5 & $0.5 \quad 1.0$ & & & & & \\
\hline & & $\begin{array}{l}\text { ver } \\
\text { gue } \\
\text { ONA }\end{array}$ & $\begin{array}{l}\text { Higher } \\
\text { fatigue } \\
\text { in IDNA }\end{array}$ & & & & & \\
\hline
\end{tabular}

Fig. 6. Meta-analysis of the cross-sectional studies using outcomes from multivariate analysis on the association between iron deficiency without anaemia (IDNA) and fatigue. ES, effect size; ID, iron deficiency; CRP, C-reactive protein. * The positive sign signifies that subjects in IDNA group complain of more fatigue than those in the non-ID group. $\dagger 1 / \mathrm{SE}^{2}$ is used as a weight. $\ddagger$ The ith study is excluded from the model-fitting. $\S$ The eight covariates used were systolic blood pressure, New York Heart Association functional class, hypertension, diabetes mellitus, efficient glomerular filtration rate, time since last heart failure admission, loop diuretics and $\mathrm{N}$-terminal pro-B-type natriuretic peptide. II Anaemic subjects were included in the multivariate analysis. If Multivariate analysis was not performed, because there were no significant differences in CRP between the two groups. ${ }^{* *}$ The three covariates were a history of suboptimal iron status, has a current medical condition and ethnicity. †† Multivariate analysis was not performed, because apparently healthy subjects were screened to exclude recurrent illness, chronic medication, anaemia, and other problems for intervention trials and characteristics were well-matched between the two groups.

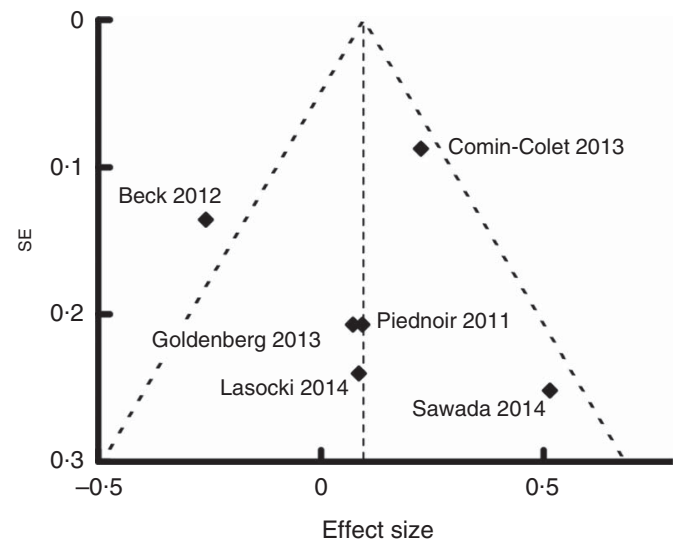

Fig. 7. Funnel plot of the meta-analysis of the cross-sectional studies using outcomes from multivariate analysis.

Several limitations should be acknowledged in this study. First, some participants with IDNA according to the authors' own definitions of Fe deficiency would be considered as having normal Fe status according to the accepted IDNA definition. There is also no established diagnostic method for IDNA in patients with inflammatory diseases. There were insufficient data to determine the critical levels of sFer or TS for selecting patients with fatigue who might benefit from an Fe preparation. Second, the association found by meta-analysis of crosssectional studies cannot infer causality. Third, although effect sizes of cross-sectional studies were corrected for possible confounders by multivariate analysis (unless study groups were screened and/or well-matched), the influence of unobserved/ unobservable confounders may still exist. Fourth, there was remarkable variation in the quality of studies, especially unbalanced sample sizes. Fifth, RCT in males could not be analysed because of the absence of relevant studies, as male cases of fatigue with IDNA are rare. Sixth, most of the cross-sectional studies were conducted in patients rather than in community settings. Finally, due to diversity in fatigue assessment methods, it was only possible to perform effect size meta-analysis.

\section{Conclusion}

In summary, our meta-analyses suggest that Fe may be effective in reducing fatigue in patients with IDNA. Further studies are now necessary to identify diagnostic criteria to select fatigue patients who might benefit from Fe treatment and to estimate the prevalence of IDNA with fatigue in the general population.

\section{Acknowledgements}

This study was partly supported by Japan Society for the Promotion of Science KAKENHI for Scientific Research (C) (grant no. 25350137).

K. Y. designed the study, conducted the search, analysed the data and wrote the manuscript; K. Y. and A. K. selected the relevant literature. All authors read and approved the final content.

The authors declare that there are no conflicts of interest.

\section{Supplementary material}

For supplementary material/s referred to in this article, please visit https://doi.org/10.1017/S0007114517001349

\section{References}

1. Public Health England and the Food Standards Agency (2014) National Diet and Nutrition Survey. Results from Years 1-4 (Combined) of the Rolling Programme (2008/2009-2011/12). London: Public Health England and the Food Standards Agency.

2. Miller EM (2014) Iron status and reproduction in US women: National Health and Nutrition Examination Survey, 1999-2006. PLOS ONE 9, e112216. 
3. Yokoi K (2014) Estimation of iron requirements for women by numerical analysis of population-based data from the National Health and Nutrition Surveys of Japan 2003-2007. J Trace Elem Med Biol 28, 453-458.

4. Lopez A, Cacoub P, Macdougall IC, et al. (2015) Iron deficiency anaemia. Lancet 387, 907-916.

5. Sydenham T (1769) The Entire Works of Dr. Thomas Sydenham, 5th ed. (J Swan, editor, English translation from Latin). London: F. Newbery.

6. Sharpe M (2006) The symptom of generalised fatigue. Pract Neurol 6, 72-77.

7. Becquerel A \& Rodier A (1844) Recherches sur la composition du sang dans l'etat de sante et dans l'etat de maladie (Research on blood in the state of health and the state of illness). Gazette Medicale de Paris 12, 751-757, 765-772, 781-802, 813-820 (in French).

8. Laache S (1883) Die Anämie. Christiania: Die Mallingsche Buchdruckerei (in German).

9. Skapinakis P, Lewis G \& Meltzer H (2000) Clarifying the relationship between unexplained chronic fatigue and psychiatric morbidity: results from a community survey in Great Britain. Am J Psychiatry 157, 1492-1498.

10. Wessely S, Chalder T, Hirsch S, et al. (1997) The prevalence and morbidity of chronic fatigue and chronic fatigue syndrome: a prospective primary care study. Am J Pubic Health 87, 1449-1455.

11. Murtagh JE (1996) Making fatigue less tiresome. Med J Aust 164, 580-581.

12. Pawlikowska T, Chalder T, Hirsch SR, et al. (1994) Population based study of fatigue and psychological distress. BMJ $\mathbf{3 0 8}$, $763-766$.

13. Beutler E, Larsh SE \& Gurney CW (1960) Iron therapy in chronically fatigued, nonanemic women: a double-blind study. Ann Int Med 52, 378-394.

14. Verdon F, Burnand B, Stubi C-LF, et al. (2003) Iron supplementation for unexplained fatigue in non-anaemic women: double blind randomised placebo controlled trial. $B M J$ 326, 1124

15. Krayenbuehl P-A, Battegay E, Breymann C, et al. (2011) Intravenous iron for the treatment of fatigue in nonanemic, premenopausal women with low serum ferritin concentration. Blood 118, 3222-3227.

16. Vaucher P, Druais PL, Waldvogel S, et al. (2012) Effect of iron supplementation on fatigue in nonanemic menstruating women with low ferritin: a randomized controlled trial. CMAJ 184, 1247-1254.

17. Morrow JJ, Dagg JH \& Goldberg A (1968) A controlled trial of iron therapy in sideropenia. Scott Med J 13, 79-83.

18. Waldvogel S, Pedrazzini B, Vaucher P, et al. (2012) Clinical evaluation of iron treatment efficiency among non-anemic but iron-deficient female blood donors: a randomized controlled trial. BMC Med 10, 8.

19. Piednoir P, Allou N, Driss F, et al. (2011) Preoperative iron deficiency increases transfusion requirements and fatigue in cardiac surgery patients: a prospective observational study. Eur J Anaesthesiol 28, 796-801.

20. Comin-Colet J, Enjuanes C, Gonzalez G, et al. (2013) Iron deficiency is a key determinant of health-related quality of life in patients with chronic heart failure regardless of anaemia status. Eur J Heart Fail 15, 1164-1172.

21. Sawada T, Konomi A \& Yokoi K (2014) Iron deficiency without anemia is associated with anger and fatigue in young Japanese women. Biol Trace Elem Res 159, 22-31.

22. Lasocki S, Chudeau N, Papet T, et al. (2014) Prevalence of iron deficiency on ICU discharge and its relation with fatigue: a multicenter prospective study. Crit Care 18, 542.
23. Goldenberg BA, Graff LA, Clara I, et al. (2013) Is iron deficiency in the absence of anemia associated with fatigue in inflammatory bowel disease? Am J Gastroenterol 108, 1392-1397.

24. Beck KL, Conlon CA, Kruger R, et al. (2012) Iron status and self-perceived health, well-being, and fatigue in female university students living in New Zealand. J Am Coll Nutr 31, 45-53.

25. Cooper H, Hedges LV \& Valentine JC (editors) (2009) The Handbook of Research Synthesis and Meta-Analysis, 2nd ed. New York, NY: Russell Sage Foundation.

26. Mullen B (1989) Advanced Basic Meta-Analysis. Hillsdale, NJ: Lawrence Erlbaum Associates, Inc.

27. Hedges LV (1981) Distribution theory for Glass's estimator of effect size and related estimators. J Edu Stat 6, 107-128.

28. Cox DR (1970) Analysis of Binary Data. New York, NY: Chapman \& Hall.

29. Sanchez-Meca J, Marin-Martinez F \& Chacon-Moscoso S (2003) Effect-size indices for dichotomized outcomes in metaanalysis. Psychol Methods 8, 448-467.

30. Haldane JBS (1956) The estimation and significance of the logarithm of a ratio of frequencies. Ann Hum Genet 20, 309-311.

31. Gart JJ \& Zweifel JR (1967) On the bias of various estimators of the logit and its variance with application to quantal bioassay. Biometrika 54, 181-187.

32. Borenstein M (2009) Effect sizes for continuous data. In The Handbook of Research Synthesis and Meta-Analysis, 2nd ed. pp. 221-235 [H Cooper, LV Hedges and JC Valentine, editors]. New York, NY: Russell Sage Foundation.

33. Weisberg S (2005) Applied Linear Regression, 3rd ed. Hoboken, NJ: John Wiley \& Sons, Inc.

34. Ferguson BJ, Skikne BS, Simpson KM, et al. (1992) Serum transferrin receptor distinguishes the anemia of chronic disease from iron deficiency anemia. J Lab Clin Med 119, 385-390.

35. Karshikoff B, Sundelin T \& Lasselin J (2017) Role of inflammation in human fatigue: relevance of multidimensional assessments and potential neuronal mechanisms. Front Immunol 8, 21.

36. Agarwal R (2007) Nonhematological benefits of iron. Am J Nephrol 27, 565-571.

37. Pratt JJ \& Khan KS (2016) Non-anaemic iron deficiency -a disease looking for recognition of diagnosis: a systematic review. Eur J Haematol 96, 618-628.

38. Peyrin-Biroulet L, Williet N \& Cacoub P (2015) Guidelines on the diagnosis and treatment of iron deficiency across indications: a systematic review. Am J Clin Nutr 102, 1585-1594.

39. Mast AE, Blinder MA, Gronowski AM, et al. (1998) Clinical utility of the soluble transferrin receptor and comparison with serum ferritin in several populations. Clin Chem 44, 45-51.

40. World Health Organization (2011) Serum Ferritin Concentrations for the Assessment of Iron Status and Iron Deficiency in Populations, WHO/nmh/nhd/mnm/11.2. Geneva: WHO.

41. da Costa BR, Rutjes AW, Johnston BC, et al. (2012) Methods to convert continuous outcomes into odds ratios of treatment response and numbers needed to treat: metaepidemiological study. Int J Epidemiol 41, 1445-1459.

42. Hasselblad V \& Hedges LV (1995) Meta-analysis of screening and diagnostic tests. Psychol Bull 117, 167-178.

43. Cruciani RA, Zhang JJ, Manola J, et al. (2012) L-carnitine supplementation for the management of fatigue in patients with cancer: an Eastern Cooperative Oncology Group phase III, randomized, double-blind, placebo-controlled trial. J Clin Oncol 30, 3864-3869. 
44. Spathis A, Fife K, Blackhall F, et al. (2014) Modafinil for the treatment of fatigue in lung cancer: results of a placebo-controlled, double-blind, randomized trial. J Clin Oncol 32, 1882-1888.

45. Brownlie T, Utermohlen V, Hinton PS, et al. (2002) Marginal iron deficiency without anemia impairs aerobic adaptation among previously untrained women. Am J Clin Nutr 75, 734-742.

46. Brutsaert TD, Hernandez-Cordero S, Rivera J, et al. (2003) Iron supplementation improves progressive fatigue resistance during dynamic knee extensor exercise in iron-depleted, nonanemic women. Am J Clin Nutr 77, 441-448.

47. Greminger AR \& Mayer-Pröschel M (2015) Identifying the threshold of iron deficiency in the central nervous system of the rat by the auditory brainstem response. ASN Neuro 7, 1759091415569911.

48. Hunt JR, Zito CA, Erjavec J, et al. (1994) Severe or marginal iron deficiency affects spontaneous physical activity in rats. Am J Clin Nutr 59, 413-418.

49. Lozoff B, Clark KM, Jing Y, et al. (2008) Dose-response relationships between iron deficiency with or without anemia and infant social-emotional behavior. J Pediatr 152, 696-702, 702 e691-693.

50. Bruner AB, Joffe A, Duggan AK, et al. (1996) Randomised study of cognitive effects of iron supplementation in nonanaemic iron-deficient adolescent girls. Lancet 348, 992-996. 